ARTIKEL 



\title{
Gewalt und Entmenschlichung in Georg K. Glasers Romanen Schluckebier und Geheimnis und Gewalt
}

\author{
Violence and Dehumanization in Georg K. Glaser's Novels \\ Schluckebier and Geheimnis und Gewalt
}

Pavel Knápek

\begin{abstract}
The article examines two of Georg K. Glaser's most famous novels, focusing on the themes of violence and dehumanization that are crucial to the works of this presently neglected author. In both books, the lives of the characters, from their early childhood, are marked by violence - not only in the family and on the streets but also in public institutions intended for the education of young people. The examples given in Glaser's works illustrate the circumstances in which victims become perpetrators and show how their lives are intertwined with the development of social and political life. Attention is also paid to human identity, human rights, and ideological systems that trample on human dignity. Glaser proves to be very good at analyzing historical, social, and psychological contexts.
\end{abstract}

\section{Keywords}

Georg K. Glaser; Schluckebier; Geheimnis und Gewalt; Secret and Violence; violence; dehumanization; German literature 


\section{Einleitung}

Obwohl Georg K. Glasers (1910-1995) Werke zweifellos viel zu bieten haben, ist ihnen innerhalb der Germanistik nur von wenigen Wissenschaftlern die gebührende Aufmerksamkeit geschenkt worden. 1966 bezeichnete Peter Härtling Geheimnis und Gewalt - den bekanntesten Roman Glasers - als eines der wichtigsten Werke des 20. Jahrhunderts, das zu Unrecht in Vergessenheit geraten sei, und initiierte dessen Neuausgabe in seiner Reihe der Vergessenen Bücher. Zuerst erschien Geheimnis und Gewalt 1951 in Französisch, erst anschließend bei mehreren Verlegern in Deutsch - allerdings oft mit Druckfehlern, in kleinen Auflagen und gekürzten Fassungen. Eingehend literaturwissenschaftlich erforscht wurden Glasers Werke erst von Michael Rohrwasser - Glasers Nachlassverwalter und Herausgeber, der sich beim Verlag Stroemfeld/ Roter Stern unter anderem um die ungekürzte Neuauflage von Geheimnis und Gewalt im Jahre 1989 (mit Nachwort und editorischen Notizen) und Schluckebier im Jahre 2007 (samt anderen Erzählungen mit einem umfangreichen Nachwort) verdient gemacht hat. Vor allem seit den späten 1980er Jahren hat die Glaser-Forschung neue Impulse bekommen, die sich vor allem in Studien über die Entwicklung der politischen Haltungen des Schriftstellers und über seine Einordnung in die Gruppe der Exil- bzw. ex-kommunistischen Autoren äußerten. Der Film Georg K. Glaser - Schriftsteller und Schmied (1988) des Dokumentaristen Harun Farocki hat wesentlich zum Verständnis der Persönlichkeit des alternden Schriftstellers und seiner Bedeutung beigetragen. Darin wird Glaser in seiner Schmiedewerkstatt porträtiert, wo er von der Bedeutung des Handwerks erzählt, das ihm gleichzeitig den Lebensunterhalt sichert, so dass er sich als Schriftsteller dem Druck der Verlage und des Buchmarkts entziehen kann. 2007 erschien Glasers erster Roman Schluckebier (1932) in Neuauflage - mit einem gehaltvollen Nachwort des Herausgebers, in dem der Fokus insbesondere auf das vielfach präsentierte Motiv der Jugendrevolte in den späten 1920er Jahren in Deutschland in einen breiten literarischen und geschichtlichen Rahmen gesetzt ist. Schluckebier beschreibt die Periode von 1918 bis 1929 aus der Perspektive eines Kindes bzw. Jugendlichen, dessen Leben stark von Demütigung, Gewalt, Nachkriegsarmut und Hunger geprägt ist und der bei der Eskalation der Revolte im Erziehungsheim durch die Polizei getötet wird.

Der vorliegende Artikel fokussiert die Problematik der Gewalt und der Entmenschlichung in den beiden ersten Romanen des Autors (Schluckebier und Geheimnis und Gewalt). Dieses Thema ist für Glasers literarisches Schaffen sehr wichtig, denn es prägt das Leben seiner Figuren bereits in ihrer frühen Kindheit. Es beeinträchtigt sie in ihrer Entwicklung und droht sie zu „Ungeheuer[n]“ (Glaser 1989: 11) zu machen, wenn ihnen keine schützende Hand zur Hilfe kommt. Die Verwahrlosten und Gepeinigten können im erwachsenen Alter dem Rausch der Macht, Rache und Gewalt verfallen. Zumindest im Fall von Valentin Haueisen (Geheimnis und Gewalt) ist es nicht dazu gekommen. In folgenden Kapiteln wird analysiert, wie sich die kindlichen bzw. jugendlichen Figuren mit der ihnen zugefügten Gewalt auseinandersetzen und wie sich dies auf ihren Charakter im erwachsenen Alter niederschlägt. 


\section{Gewalt}

Glasers erster Roman Schluckebier erschien 1932 als das Werk eines 21-jährigen Journalisten und beginnenden Schriftstellers, der zu jener Zeit schon seit fünf Jahren den kommunistischen Jugendorganisationen sowie dem BPRS (Bund proletarisch-revolutionärer Schriftsteller) angehörte. In Frankfurt am Main wurde er zum Gerichtskorrespondenten bei der kommunistischen Arbeiter-Zeitung, doch seine Reportagen erschienen auch bei der renommierten Frankfurter Zeitung. Der Ausweis des Gerichtsreporters ermöglichte Glaser den Zutritt in den Gerichtssaal, wo er Zeuge der Schilderung von Verbrechen, menschlichen Tragödien und verheerenden Urteilen wurde. Der Autor, selbst kurz zuvor wegen Gewalt gegen Polizisten für sechs Monate zur Gefängnisstrafe verurteilt, musste seine Arbeit des politisch vereinnahmten Berichterstatters als zwiespältig empfunden haben. Dem späteren Roman Geheimnis und Gewalt zufolge wurde vom Journalisten eines kommunistischen Blattes die Einnahme ideologischer Standpunkte gefordert, die erstrebenswerte Versuche um tiefere Einsichten in die Situation und Motive der Beteiligten in vielen Fällen verhinderte, besonders wenn es um politische Gegner ging. Der autodiegetische Erzähler in Geheimnis und Gewalt in der Rolle des Gerichtsreporters erzählt:

„Ich berichtete über den Mordprozeß ,Stubenrauch und Genossen'. Es waren drei Braunhemden, der Älteste und Hauptschuldige zwanzig, die beiden Helfer jeder 18 Jahre alt. [...] Traurig, aber auch wie ein Soldat [...] setzte ich unter der Aufsicht unseres Schriftleiters auf: ,Drei viehische braune Mordbuben, Abschaum des Lumpenproletariats.““(Glaser 1989: 114-115)

Michael Rohrwasser vermutet in diesem Gewissenskonflikt der Hauptfigur den Anstoß zur Niederschrift von Schluckebier (vgl. Rohrwasser 2007: 330). Dieser frühe Roman beschreibt die traurige Kindheit und Jugend eines Knaben aus ärmlichen Verhältnissen mit einem gewalttätigen Vater und setzt sich im hohen Maße mit der Thematik der Gewalt und deren Voraussetzungen auseinander. Der Lebensweg des Protagonisten führt von Zuhause auf die Straße; von der Schule ins Gefängnis und in die Erziehungsanstalt „Billigheim“, wo er schließlich bei der Niederschlagung des Zöglingsaufstands durch Polizisten erschossen wird. Das Werk erschien 1932 in dem der KPD nahestehenden Agis-Verlag. Wie der Rezensent Siegfried Krakauer richtig bemerkt, geht es dem jungen Verfasser um keine bloß dokumentarische Festhaltung von schockierenden Bedingungen, die die Erziehung des Jungen prägten. Der gleiche Rezensent schreibt über Glasers Roman auch folgendes: „Statt die Tatsachen sprechen zu lassen, spricht Glaser lieber selber“ (Krakauer 1985: 150-151). Der Satz ist durchaus kritisch gemeint, aber es zeigt sich, dass im Hinblick auf das geschilderte Thema der Misshandlung von Kindern und Jugendlichen die Strategie der analysierenden Stimme des Erzählers gut gewählt ist, da die verheerende Wirkung der Gewalt durch die kindliche Perspektive umso deutlicher hervortritt. Die Misshandlung durch den Schutzmann, der das hungrige Kind auf den Kopf schlug, als es Zuckerrüben - „eigentlich [...] Viehfutter“ (Glaser 2007: 102) - gestohlen hatte, wurde für Schluckebier zum initiierenden Prozess der Entwürdigung, Verstörung und Empörung gegen die Gesellschaft. Später hatte der heranwachsande Junge 
unter den brutalen Schlägen seines Vaters zu leiden, der oft bei geringsten Anlässen „einfach drauflos schlug“ (Glaser 2007: 113). Der Erzähler neigt dazu, die Perspektive des Kindes zu übernehmen, das die Gewalterfahrung zu verarbeiten versucht, aber an deren Unerklärlichkeit immer wieder scheitert: „So oft er an die Tat des Schutzmannes dachte, ließ das Unbegreifen alles in ihm stillstehen. Er stand wie vor einer Mauer und träumte sich in eine wütende Empörung hinein." (Glaser 2007: 103) Das Unvermögen des Verstehens der Gewalt rief in Schluckebier eine große Verbitterung und Empörung hervor, die in Hass mündete, aber zugleich „eine große Übelkeit in [Schluckebiers] kindlichem Körper“ (Glaser 2007: 106) hervorrief. An unzähligen Stellen im Roman werden Hass und seine Wirkung analysiert, denn die Hauptfigur gerät immer wieder in Situationen, die dieses Gefühl in seinem Inneren hervorrufen. Der Roman belehrt darüber, dass Gewalt erst dann „verstanden“ werden kann, wenn man sie selbst begeht. Dann aber entwickelt sie sich zu einer verheerenden Spirale, die immer weiter und mächtiger um sich greifen kann. Das zeigt vor allem der zweite Teil des Romans, in dem Schluckebier zusammen mit den übrigen Zöglingen der extremen Brutalität des „Hausvaters“ im Erziehungsheim ausgesetzt ist. Dieser ging so weit, dass er ihren kranken Kameraden einsperren und schließlich verhungern ließ. Dieses Ereignis wurde zum Auslöser des gewalttätigen Aufstands gegen die gesamte Leitung des Billigheims. Die aufständischen Jugendlichen können jedoch nicht mit voller Sympathie betrachtet werden, da sie mindestens in einem Fall - wie sich später zeigt - einen völlig ungerechten Mord begehen. Dies geschieht nach der Demütigung und Verjagung des Direktors und aller Erzieher. Die Zöglinge, aus deren Perspektive („Wir“) im zweiten Teil überwiegend erzählt wird, verletzen tödlich einen unbekannten Flüchtenden, in dem sie instinktiv - aber ohne jeden Beweis - den berüchtigten Hausvater Albert (Drecksack genannt) vermuten:

„Ein Mensch in grünem Anzug kam zu Vorschein. Wir hatten alle einen Gedanken. Wir rasten über das Pflaster auf den grünen Loden zu. Der verschwand. [...] Auf der Leiter, die zum Dach führte, stand der Grüne. Er streckte uns die Hände entgegen, wir glaubten einen Augenblick, daß es gar nicht Drecksack war. Aber gerade, daß er uns so flehend die Hände entgegenstreckte, erinnerte uns an die Schlafsaalszene mit Otter. [...] Ein Junge holte mit der Axt aus und schlug nach oben. Er traf den Fliehenden in das rechte Bein. Wir sahen, wie er zuckte, fest in die Sprossen griff und in Todesangst die Leiter hinaufstrebte. [...] Der Mann war schwer verwundet, er hielt kaum den Oberkörper aufrecht. Wieder wollten wir aufhören oder schnell Schluß machen. Einige Jungens liefen mit Aexten über das Dach; der Mann verstärkte sein Flehen; wir fingen an zu zögern, aber in demselben Moment schwankte er und verschwand. [...] Wir hatten ein schlechtes Gewissen." (Glaser 2007: 225)

Um das alarmierende Moment des Verbrechens zu unterstreichen, wird zwei Absätze weiter über den plötzlich aufgetauchten Hausvater berichtet, der nicht getötet wurde. Der tote unbekannte Körper tritt bis zum Ende des Romans noch dreimal in Erscheinung. Es wird über ihn auf unterschiedliche Art und Weise berichtet: „Ganz vorn, direkt hinter dem Haus lag ein grüner Knäuel. Wie auf Verabredung fragte niemand danach.“ (Glaser 2007: 230) An anderer Stelle sprechen die Jungen über ihn als über 
einen Ballen Heu, toten Hund, Matratze oder toten Körper (vgl. Glaser 2007: 231). Fast am Ende der Handlung, nach Schluckebiers Tod und nach der Verhaftung der übrigen Insassen lesen wir folgende Sätze, die - in einen Absatz eher zusammenhanglos eingeschoben - zynisch ausklingen: „In der Ecke lag eine Plandecke über einem länglichen, mannsgroßen Etwas. Die Decke hatte einige große rote Tupfen." (Glaser 2007: 240) Aus dem Text geht hervor, dass es sich in diesem Fall um die Leiche des verhassten Hausvaters (Drecksack) handelt, den die Zöglinge tödlich verwundet haben. Die Perspektive des „Wir“-Erzählers - d.h. der von der Polizei umzingelten Rebellen - steigert sich am Ende zur hemmungslosen Aggression. Nach der Tötung Drecksacks angesichts des drohenden polizeilichen Angriffs heißt es nun: „Wir rannten fast fröhlich. Freier als gestern abend - jetzt war die Rechnung glatt, und es konnte uns nichts mehr überraschen." (Glaser 2007: 235) In der Phase nach den begangenen Gewalttaten und in der Bedrohung durch die Todesschüsse der Polizei wird die eigene Schuld nicht mehr wahrgenommen. Die oben erwähnte Übelkeit des kleinen Schluckebier über die eigenen Hassgefühle verspüren die Zöglinge nicht mehr. Die tyrannisierten Jungen haben sich die brutale Handlungsweise zu eigen gemacht. Die Gewaltspirale hat um sich gegriffen und wird sich weiter verbreiten.

Verantwortlich für diese Entwicklung wird (an mehreren Stellen des Textes) die „Prügelpädagogik" gemacht. In diesem Sinne ist Schluckebier ein Anti-Gewalt-Roman, indem er die verheerenden Folgen der Gewalt an den Jugendlichen zeigt und ihre Gefahr für die Gesellschaft deutlich macht. Auf der expliziten Erzähler-Ebene ist das Buch aber auch rebellisch-kommunistisch, indem es keine friedliche Lösung für die ausgebeutete Arbeiterklasse in Aussicht stellt und die Straßenkinder, die von Anfang an mit Hunger und Unterdrückung konfrontiert werden, zu Gewalttätern erzieht. Implizit macht das Buch auf die drohende Gefahr der Gewaltentzündung durch eine - anscheinend kommunistische - Revolution aufmerksam, wie noch gezeigt wird.

Den Darstellungen in Schluckebier zufolge hat die Prügelpädagogik der Väter bzw. Erzieher ihre Grundlage im „Neid und Haß und Ehrgeiz“ (Glaser 2007: 118) an Arbeitsplätzen sowie im Geiz der Reichen und in ihrer Angst, ihre Posten bzw. ihre Besitztümer zu verlieren. Schluckebier beschreibt die Periode vom Ende des Ersten Weltkriegs an bis in die späten 1920er Jahre in Deutschland, die von bitterster Armut und politischer Gewalt geprägt waren. Am Ende des Romans wird der Ausbruch der Weltwirtschaftskrise angedeutet. Die Zöglinge in Billigheim dienen als billige Arbeitskraft und ihre Verpflegung entspricht nicht den Mindeststandards. Diese Tatsache wird expressiv ausgedrückt: „Angst um Posten. Es ist alles, was sie sagen: Ehre, Erziehungsreform, Zucht, Pflicht auf einen gemeinsamen Nenner zu bringen: Fressen, Posten." (Glaser 2007: 207) Viele Insassen des Billigheims arbeiteten vor ihrer Einweisung in der sogenannten Rotfabrik - dem chemischen Betrieb, wo sie schutzlos mit giftigen Stoffen umgehen mussten, so dass die meisten von ihnen dies mit ihrer Gesundheit oder gar mit ihrem Leben bezahlten. Die kommunistischen Forderungen nach menschenwürdiger Behandlung und Bezahlung sind in diesem Fall voll berechtigt. Die Parolen der Kommunisten bei Demonstrationen wie „Hunger“ (Glaser 2007: 145) oder „Gebt uns Arbeit und Brot, sonst schlagen wir euch tot“ (Glaser 2007: 142) scheinen die Zustimmung des Erzählers zu haben: 
„Die Kommunisten sangen, daß sie für ein täglich Stück Brot kämpften. Daß jeder hat sein täglich Stückchen Brot. [...] Und diese Forderung war doch so illusionslos, so nüchtern. In der Schule hatten sie gesungen, Feiern abgehalten, die Lehrer und Pfarrer hatten geredet, für die hohen Ideale, für Gott, für Kultur, für Vaterland; lauter hohe, geheimnisvolle Dinge. Und doch hatte hinter ihnen so wenig gesteckt, daß man mit ihnen nicht vor dem Leben bestand.“ (Glaser 2007:133)

Die Parteigenossen werden im Schluckebier zunächst etwas idealisiert dargestellt - kameradschaftlich, selbstlos, zuweilen heroisch. Ihre Demonstration, die im Roman geschildert wird, wurde durch die polizeiliche Gewalt brutal niedergeschlagen. Der Schluss von Schluckebier kann jedoch als die Vorbereitung einer gewalttätigen kommunistischen Revolution gelesen werden. Nachdem Schluckebier und andere drei Zöglinge von der Polizei getötet wurden, wird die Situation aus der „Wir“-Perspektive der übrigen aufständischen Zöglinge geschildert. Die Schlussszenen entbehren nicht eines gewissen revolutionären Pathos:

„Die Revolte lebte noch. Sie tickte gesund, wie das Herz in uns. [...] Wir standen stramm. Hände an der Hosennaht. Vorschriftsmäßig. Hinter uns lag das Ried und Selbold und die Rotfabrik ${ }^{1}$. Das war uns, als ständen die Arbeiter aus Selbold, die Bauern aus dem Ried und die Rotfabriker selbst hinter uns. Wir waren nur die Spitze eines gewaltigen Zuges." (Glaser 2007: 240)

Die Zöglinge sind zur Revolte, ja zur Revolution, bereit: „Unsere Hände krümmen sich langsam zu Fäusten. Jetzt kann es losgehen. Wir stehen, Fäuste an der Hosennaht. Die ganze Welt steht Fäuste an der Hosennaht hinter uns. Uns ist der Rücken gedeckt. Wir haben nichts zu verlieren. Wollt ihr was?“ (Glaser 2007: 240) In möglicher Assoziierung an den „Westen“ (d.h. „die alte Welt“) ist am Ende von Schluckebier die Rede vom „leise[n] Westwind“, der nach der Rotfabrik riecht (Glaser 2007: 239). „Es ist ein Geruch von Gift und Aufruhr“ (vgl. ebd.). Wie bereits gezeigt, handelt es sich dabei um keine unreflektierte Glorifizierung der kommenden Revolution, die die Gefahren der Gewaltspirale nicht zumindest andeuten würde. Die Revolte der überlebten Zöglinge ist bereits durch die von ihnen begangene Gewalt befleckt - den Mord an einem Unbekannten. In seinem Nachwort (2007) zu Schluckebier macht Michael Rohrwasser mit Recht darauf aufmerksam, dass die pathetisch prophezeite Revolution der Zöglinge zu befürchten sei. Sie würde nämlich von Menschen geführt werden, deren Charakter von Gewalt und Entmenschlichung geformt wurden. Diese Menschen haben hassen gelernt: „Glasers Erzählung berichtet von einer Angst, die ins Innere greift. Die Bremse des Zuges, die sich löst,

1 Die berüchtigte Fabrik, in der Giftstoffe (unter anderem für die Rüstungsindustrie) produziert wurden. Die meisten dortigen Arbeiter trugen dauerhafte schwere Schäden davon. Viele sind umgekommen. Der Name rührt von der Farbe der Hände vieler Arbeiter, die dort angestellt waren. Die Rotfabrik ist nicht lokalisierbar, aber sie erinnert an die Farbwerke Hoechst bei Frankfurt am Main, die zum I.G.-Farben-Komplex gehörten. Dort hat auch Georg K. Glaser in seiner Jugend unter schrecklichen Bedingungen gearbeitet (vgl. Rohrwasser 2007: 346). 
die Ventile des Dampfkessels, die sich nicht mehr öffnen lassen, lösen den Schrecken aus vor dem, was hier freigesetzt wird, einer bête humaine, geboren in den Fabriken und Fürsorgeheimen." (Rohrwasser 2007: 346-347) Allerdings stellt das revolutionäre Pathos am Ende des Buches - trotz aller Gewalt - eine logische Reaktion der Jungen dar.

Eine indirekte Kommunismuskritik zeigt Georg K. Glaser bereits zwei Jahre nach der Herausgabe von Schluckebier (1932) in seiner Meisternovelle Die Nummer Eins der Rotfabrik (1934). Impliziert kritisiert sein Text die Bestrebungen einer kommunistischen Zelle um die Verteilung ihres Blattes im nationalsozialistischen Deutschland. Diese Aktion setzt nicht nur das Leben der tatsächlichen Autoren aufs Spiel. Die Tat erscheint im Endeffekt als sinnlos und marginal. Die Rache der Nazis trifft nämlich einen Arbeiter, der - unabhängig von der Tat der Kommunisten - sich offiziell weigerte, eine Gruppe von jungen Arbeiterinnen (einschließlich seiner Braut) in jene berüchtigte Abteilung des Betriebs zu schicken, wo mit lebensgefährlichen Kampfstoffen ohne wirksame Schutzmittel gearbeitet wurde (vgl. Rohrwasser 1991: 246-248). Glasers Kritik am Kommunismus geht noch viel weiter in den Werken, die nach dem Zweiten Weltkrieg erschienen. Dort wirft er den kommunistischen Zentralen vor, durch die Befehle in ihren Direktiven die Leben der Parteigenossen wegen sinnloser Aktionen gefährdet zu haben. Ähnlich wie die Arbeiter unter lebensgefährdenden Situationen in der kapitalistischen Rüstungsindustrie arbeiten mussten und faktisch zu „Produktionsmitteln“ degradiert wurden, war es der Fall auch in der hierarchisch geleiteten Kommunistischen Partei. Der Prozess der Entmenschlichung mittels Gewalt hat sich hier fortgesetzt. Der Zynismus der kommunistischen Direktiven wird noch an anderen Beispielen am Ende des folgenden Kapitels gezeigt.

\section{Entmenschlichung}

Georg K. Glaser zeigt, dass die Gewalt besonders dort gut gedeiht, wo ihren Opfern der Status der bedingungslosen menschlichen Würde - explizit oder implizit - abgesprochen wird. Eine solche Gewalt wird vordergründig von Strukturen mit Machtstatus ausgeübt. In Schluckebier handelt es sich vor allem um: Lehrer, Erzieher, Polizei, Arbeitgeber oder den eigenen Vater. Andererseits können sich unterdrückte Personen unter Umständen zu kollektiven Strukturen (z.B. Schüler, Zöglinge) vereinigen, die als Masse hemmungsfrei und gewalttätig handeln können. Glasers Schluckebier zeigt solche gewaltunterstützenden Mechanismen beider Art an vielen Beispielen. Die Gewalt geht in solchen Fällen mit dem Entmenschlichungsprozess einher. Die Entmenschlichung hat mehrere Erscheinungsformen. Allen gemeinsam ist die Schaffung oder Vertiefung von (künstlichen) Barrieren zwischen den sich gegenüberstehenden Subjekten mit dem Ziel der Wahrnehmung des anderen als gefährlich oder minderwertig. Die Formen sind unterschiedlich: Verspottung und Verleumdung; möglichst komplizierte Bürokratisierung und Automatisierung (in Industrie und Wirtschaft); hoher Grad von Hierarchisierung; Vereinnahmung der Sprache - Ideologisierung des Sprachgebrauchs; möglichst abstrakte Ausdrucksweise oder Anspruch auf absolute Wahrheit (Ideologie, evtl. Religion). 
Der Verspottung und Verleumdung wird Schluckebier vor allem in der Schule ausgeliefert. Im Rahmen eines Integrationsbestrebens wird er in eine Schule für Knaben aus wohlhabenden Familien aufgenommen. Von diesen unterscheidet er sich jedoch durch seine ärmliche Kleidung und dadurch, dass er keine Schuhe hat, was zum Grund für seine Verspottung und für Gewalt gegen ihn wird - ebenfalls vonseiten der Lehrer.

„Seine Mitschüler schlugen ihn jeden Tag. Sie traten ihm auf die Barfüße. Die Lehrer riefen ihn immer in den Stunden vor die Tafel, weil seine nackten Füße auf dem Boden so klatschten. Die Klasse lachte immer und der Lehrer hielt das Herausrufen für einen guten Witz. [...] In den Stunden schlugen die Lehrer. Sie drehten die Haare herum, oder zogen an den Ohren. Sie gaben Ohrfeigen; Stockschläge auf den Rücken, Kopf und Hände. Sie erwarben sich einen traurigen Respekt, wenn sie mit den Jahren eine besonders schöne Art des Wehetuns herausarbeiteten." (Glaser 2007: 113)

Der Versuch einer von oben angeordneten Eingliederung des Schülers ins Kollektiv der wohlhabenden Sozialschicht bleibt nicht nur erfolglos, sondern befestigt und vergrößert die bereits vorhandene Klassenkluft. Außerdem wird der Schüler Schluckebier im Schulmilieu mit den ideologisch bedingten und als Lüge entlarvten Narrativen konfrontiert. Er bemerkt, dass die Lehren, die dort vermittelt werden, dazu bestimmt sind, die Klassenunterschiede zu begründen und zu befestigen. Zum Beispiel hört er davon, dass „alles Gottes Fügung [sei] und als solches hingenommen werden müsse“ (Glaser 2007: 139). Die Erklärungen der Lehrer erscheinen dem heranwachsenden Schluckebier absurd:

„[Die Lehrer] erzählten auf jede Warum-Frage eine verlegene Geschichte. [...] Sie machten lächerliche Versuche zu antworten. Während in der Welt alles in die Brüche ging, und die Meute Kohldampf zum Platzen schob, und nicht wusste wohin, machten sie unverfroren ihren alten Salat weiter. Der Pfarrer erzählte immer noch von Sünde und vom Falle des Satans. Die Alten drohten immer noch mit dem Finger. [...] ,Die Jugend muß wieder glauben lernen. Der Glaube wird sie aufrecht erhalten.““(Glaser 2007: 140)

Der Gebrauch von Glaubenssätzen in Funktion von Argumenten vermischt mit impliziten Drohungen (Verweise auf „Sünde“ oder „Hölle“) schaffen eine Kommunikationsbarriere, die die brennenden Fragen und die Gesellschaftskritik im Keim erstickt. Das „materialistische“ Denken wird mit Hilfe der Religionsideologie von vornherein abgewiesen. Indem die Fragen und Argumente der hungernden Jugendlichen als profan und niedrig abgewertet werden, findet eine Art Entmenschlichung statt, die die benachteiligten Heranwachsenden als „der Sünde verfallen“, oder zumindest unreif ausschließt. Auf der praktischen Alltagsebene geht es jedoch darum, dass die kirchlichen Wohltätigkeitsorganisationen zu machtlos oder teilweise unwillig sind, der unüberschaubaren Menge der Armen, die nötige Hilfe zu leisten oder sich für ihre Rechte lautstark einzusetzen. ${ }^{2}$

2 "Man war schon in den Klöstern bekannt, die Schwester Pförtnerin zählte schon, wie oft man schon zu viel kam, und der Heilsarmee kostete das Essen Geld, denn auch Uniformen kosteten Geld, und das Jugend- 
In Deutschland nach dem Ersten Weltkrieg herrschten erhebliche Vorurteile den Proletariern gegenüber. Vor allem wurden ihnen Atheismus, Vaterlandslosigkeit und Promiskuität angelastet. Otto Rühle (1922) macht auf die katastrophalen Wohnverhältnisse der Arbeiterklasse aufmerksam. In den meisten Arbeiterwohnungen hätten so viele Menschen gelebt, dass Zimmer und Betten von mehreren Personen geteilt werden mussten. Angesichts dieser Situation wurden in den Arbeiterfamilien anstößige Verhältnisse vermutet. Selbstverständlich begünstigte die finanzielle Not die Prostitution und es wurden viele uneheliche Kinder geboren. Detailliert werden die Probleme des Proletariats in zahlreichen Publikationen von Otto Rühle dargelegt. Klaus Theweleit (Männerphantasien, 1977/8) vermutet in den Stereotypen der Bürgerlichen über die Ruchlosigkeit und Tücke der „roten“ Proletarierfrauen die grundlegende psychologische Ursache für den Hass der militanten Nationalisten (z.B. Freikorpssoldaten) gegenüber den Proletariern, der unter anderem in die Massaker an den „Roten“ unmittelbar nach dem Ersten Weltkrieg ausartete. ${ }^{3}$

Äußerst kritisch erscheint in Schluckebier die Situation der Arbeiter in vielen Industriebetrieben. Ganz ihrer menschlichen Würde beraubt sind die Arbeiter einer Fabrik, wo sie der entpersönlichten Macht ihrer Betriebsleitung völlig wehrlos gegenüberstehen. In diesem Zusammenhang kann die Einführung der Fließbandarbeit als ein anschauliches Bild der ohnehin entmenschlichten Verhältnisse zwischen Arbeitgebern und -nehmern erwähnt werden. Die Einstellung der Arbeiter auf das Hetztempo des maschinellen Fließbands macht sie noch mehr zu Automaten und jeder Fehler kann schreckliche Folgen nach sich ziehen: „Heute früh wurden einem der achtunddreißig Lehrlinge der Werkzeugmacherei drei Finger der rechten Hand und, als er wahnsinnig mit der anderen zugriff, die ganze linke Hand abgerissen. Allen sichtbar hing die eigentliche Ursache der nervösen Unruhe und der Unfälle überall: das neue Fließband.“ (Glaser 2007: 129) Allerdings ist das Fließband ein von Menschen konstruiertes Instrument, dessen Erfindung und Einstellung auf das schnelle Tempo nur Menschen (Auftraggebern) zulasten gelegt werden kann. Die ausbeutenden Besitzer verstecken sich hinter den Maschinen, durch deren Einsatz sie die höchstmögliche Arbeitseffizienz verlangen. Diese Maßnahme der extremen Entmenschlichung ist dazu geschaffen, den direkten Kontakt zwischen Arbeitgebern und -nehmern zu verhindern.

Nicht nur Menschen im Besitz der Macht können sich die Entmenschlichungsmechanismen zunutze machen. Ebenfalls Bevölkerungsgruppen, die sich benachteiligt fühlen und aufgrund ihrer gemeinsamen Bedürfnisse sich zu einer organisierten oder unorganisierten Masse zusammenschließen, um ihre Interessen durchzusetzen, neigen zum Streben nach dem größtmöglichen Machtanteil. Dabei können sie sich der Entmenschlichung ihrer Gegner als eines geeigneten Instruments bedienen. In Schluckebier geschieht dies insbesondere im zweiten Teil des Romans, der das Leben der Zöglinge

asyl nahm einen nur zwei Tage auf, und seit 24 Stunden hatte man Hunger, und schon vorher hatte man nur schlechte Herz-Jesu-Suppen gehabt, dann tat man, was einem leichter fiel: Klauen oder Betteln; beides wurde bestraft." (Glaser 2007: 138)

3 Theweleit stützt sich auf Briefe und Schriften der deutschen Freikorpssoldaten im Ruhrgebiet und im Baltikum. 
im Erziehungsheim („Billigheim“) schildert. Die Jungen wurden hier Zeugen von sadistischer und verbrecherischer Brutalität seitens mancher Erzieher - besonders des Hausvaters (genannt Drecksack, vgl. den Abschnitt „Gewalt“ im vorliegenden Beitrag). Nach dem von Drecksack verursachten Hungertod ihres Kameraden sind in Billigheim so gut wie alle Hemmungen der Zöglinge gefallen. Allen Lehrern und Erziehern wurde der Gehorsam verweigert. Viele der „Pädagogen“ wurden gedemütigt, verprügelt oder gar umgebracht (der fliehende Unbekannte und der Hausvater am Schluss). Der Prozess der Entmenschlichung wird in Schluckebier in direkten Zusammenhang mit der Einordnung des Einzelnen in die Masse gebracht. Die Unterstellung des Menschen unter die Masse erscheint als dessen Befreiung von der eigenen Verantwortung. Im Zusammenhang mit der gewaltsamen Revolte im Billigheim lesen wir: „Wenn jetzt jemand etwas selbständig getan hätte, so wäre ihm die Verantwortung dafür zugefallen. Nur indem man in der Masse blieb, war man vor der Last der Verantwortung sicher." (Glaser 2007: 209) Der Teufelskreis der Gewalt wird durch den folgenden Satz treffend ausgedrückt: „Auf Drecksack hatten alle geschlagen, weil alle geschlagen hatten." (ebd.)

Die Kommunisten, zu denen Georg K. Glaser selbst gehört hatte und von denen er sich später trennte, bilden einen Sonderfall jener Gruppierungen der Unterdrückten, die sich versammelten, um ihre zum Teil berechtigten Forderungen durchzusetzen. In den meisten Gesellschaften, in denen sie siegreich wurden, etablierten sie jedoch eigene Eliten, die in der Regel zu Unterdrückern wurden. Glasers bekanntester Roman Geheimnis und Gewalt beschreibt am Ende die zynische Kehrtwendung der kommunistischen Zentrale, die ihre Direktiven an einfache Parteigenossen weitergab und die für diese verbindlich waren. Die Hauptfigur des Romans - der deutsche Kommunist Valentin Haueisen, der vor den Nazis nach Frankreich geflohen ist, um letztlich doch als französischer Soldat (unerkannt) in deutsche Gefangenschaft zu geraten, erfährt den Wortlaut jener kommunistischen Direktive, die die Reste seiner Hoffnungen auf die „Partei“ endgültig begrub. Der lange erwartete Befehl der Partei lautete: „Tuez les Boches.“ (Glaser 1989: 480) (d.h.: „Erschlagt die Deutschen.“) Glasers Roman deutet an, dass es sich bei dieser Direktive an die französischen Kommunisten um ein gut überlegtes Kalkül handelte, mit dem Ziel den Hass der Franzosen gegen Deutsche für die Zwecke der kommunistischen Revolution auszunutzen. Es ist ein zynischer Gebrauch des Kollektivhasses, vor dem sich die Kommunisten im Roman nicht scheuten, obwohl er im direkten Widerspruch zu ihrer Lehre stand, nach der sich die Proletarier aller Länder vereinigen sollten, um die herrschende Klasse auf internationaler Ebene zu besiegen. Der Klassenkampf war für sie die „Lösung“ gewesen, nicht der Völkerkampf.

Der bekannteste Fall des Kollektivhasses und der beispiellosen Entmenschlichung, die in das größte Massenmorden aufgrund der Rassenzugehörigkeit ausartete, war der Holocaust. Glaser zeigt die Verinnerlichung der pervertierten Ideologie der Nationalsozialisten am Beispiel eines jungen Soldaten, der mit Morden an Juden prahlt:

„,Ich habe neunundzwanzig Juden erschossen‘. [Der Erzähler:] Ich sah fassungslos in das Kindergesicht - [...] Ich konnte mit Mühe meinen Abscheu und Zorn in Worte dämmen: ,Und die Schande erstickt dich nicht? Du bist kein Soldat, du bist ein Mörder‘ [...] Und während er 
versuchte, mich zu bekehren, einfältig glaubend, freudig zeugend, sahen mich aus ihm hunderttausend der jungen Verelendeten aus der Zeit vor dem Abenteuer an. Es waren wenige darunter, die nicht geträumt und inbrünstig davon geschwärmt hatten, die nicht fähig und bereit gewesen waren, neunundzwanzig Junker, Pfaffen oder Bürger [...] aufzuhängen, eigenhändig, im fröhlichen Bewusstsein, eine neue saubere Welt damit vorzubereiten." (Glaser 1989: 439-40)

Nach dem anfänglichen Entsetzen über die Worte des Jugendlichen findet es Glasers Erzähler doch unehrlich, den Mörder zu verdammen. Die Antwort des Textes scheint darin zu liegen, dass die Gewalt, die der junge Unbekannte ebenfalls im Elternhaus, im Heim oder Gefängnis erlitten haben mag, die Erklärung sein kann. Das erlittene Unrecht kann neue Gewalt auslösen - besonders wenn dieser Prozess von den nationalsozialistischen „Gewaltschleichern“ unterstützt wurde. Dem Motiv der misshandelten und zur Rache bereiten Jugend begegnen wir in der Erziehungsanstalt in Schluckebier, wo die Wirkung der Gewalt explizit beschrieben wird:

„In ihren Augen saß etwas Seltsames. Wenn man es das erste Mal bemerkte, fiel es nicht weiter auf. Aber später erschrak man. Tierische Rohheit, Brutalität; unendlicher, nicht faßbarer Haß; all diese Worte reichen nicht aus, um das nur annährend zu treffen, was jeden still werden ließ, der diese Augen im richtigen Winkel gesehen hatte." (Glaser 2007: 164)

Geheimnis und Gewalt scheint hier anzuknüpfen, indem es die Opfer von damals mit den Tätern der Zukunft identifiziert. Aus dem Heer der ehemals Verwahrlosten und Misshandelten sei „das ,nackte Schwert der [pervertierten] Revolution“ [geworden;] nur gegen wirkliche Kugeln nicht gefeit“ (Glaser 1989: 442) - allerdings mit ideologischer Indoktrination ausgerüstet.

Am Ende des Romans berührt der Erzähler die Problematik der Kollektivschuld der Deutschen im Zweiten Weltkrieg, wenn er schreibt: „Wie groß war die Versuchung zu glauben, daß nur ihre Ausrottung [...] sie aus der Welt schaffen konnte; so sehr fordert der Mord den Mord heraus." (ebd.) Der Erzähler - Deutscher und zugleich das Opfer des Nationalsozialismus - kann einerseits den Rachedurst der Opfer, ja sogar ihre Mordlust verstehen. Trotzdem überwiegt in Geheimnis und Gewalt die tiefe Enttäuschung über den Kollektivhass gegenüber Deutschen nach dem Zweiten Weltkrieg. Das Konstrukt der deutschen Gesamtschuld betrachtet der Erzähler als die Fortsetzung des „Werk[s] der [nationalsozialistischen] Verbrecher [-], die seit elf Jahren dafür sorgten, das deutsche Volk in seiner Gesamtheit als tatsächlichen Urheber gelten zu lassen." (Glaser 1989: 389) Glasers Erzähler als ideologischer Gegner der Nazis, dessen zahlreiche deutsche Kameraden von dem verbrecherischen Regime ermordet wurden, findet keinerlei Sympathie für die Perspektive des Hasses gegen die Deutschen. Der Kollektivhass und die Kollektivschuldzuweisungen betrachtet er als die gefährlichsten Formen der Entmenschlichung. Durch sie erzeugt der abstrakte Hass extreme Gewalt, welche wiederum neues Unrecht heraufbeschwört. Valentin Haueisen in Geheimnis und Gewalt - und mit ihm der Autor selbst - haben ein neues Europa ersehnt, das aus den alten Fehlern zu lernen imstande wäre. Dies wird im folgenden Bild zum Ausdruck gebracht: 
„das Bild einer Zukunft, einer Friedenszeit, in der das Vaterland der Werktätigen und die Partei die Meinungsfreiheit wiederhergestellt - hatten sie nicht die Kirchen wieder erlaubt? - einer Zukunft, in der die Kirche umgekehrt um die gerechte Verteilung der Güter focht, in der die Sieger sich als Erzieher und Befreier über die Besiegten beugten“. (Glaser 1989: 471)

Dieser Traum ist zum Zeitpunkt der Niederschrift des Romans (1945-48) noch nicht in Erfüllung gegangen. „Das ,Erschlagt die Boches““ [...] hatte gesiegt über das „freie Europa gegen das braune Europa“, schreibt der Autor. (Glaser 1989: 496-7)

\section{Fazit}

Der vorliegende Beitrag hat zunächst das Thema der Gewalt in Georg K. Glasers Schluckebier analysiert. In diesem Roman wird unter anderem die allgegenwärtige Brutalität im Erziehungsheim geschildert, in das die jugendliche Hauptfigur eingewiesen ist. Ebenfalls in Geheimnis und Gewalt wird das Leben in einer solchen Anstalt dargestellt, wenn auch teilweise anders und weniger drastisch. Glasers Werke zeigen eindrücklich die verhängnisvolle Wirkung der Gewalt im Erziehungsprozess. Diese wird in den Werken des Autors als Auslöser von Angst- und Hassgefühlen im Menschen identifiziert. Dieser psychologische Prozess setzt Selbstverteidigungsmechanismen im jungen Menschen in Gang, die den Anfeindungen der Umgebung mittels der Entwicklung eines eigenen ständigen Hassgefühls gegenüber der menschlichen Gesellschaft trotzen. Indem der Erzähler von Schluckebier meistens die Perspektive der Hauptfigur übernimmt und ihre Gedankengänge den Lesern vermittelt, wird der Prozess der Erziehung zur Gewalt anschaulich demonstriert. Obwohl Glasers Romane, die hier analysiert werden, fiktional und nicht voll autobiographisch sind, tragen sie im hohen Maße zum Verständnis der deutschen Geschichte zwischen 1918 und 1945 bei. Ihr Verdienst ist auch, dass sie die Perspektive der niedrigsten Sozialschicht zur Geltung bringen, wobei sie keiner Romantisierung oder Ideologisierung unterliegen. Glaser zeigt in seinem Werk, wie die jungen Verwahrlosten zur Zielscheibe von Ideologen verschiedenster Richtungen werden. Sie sind für neue Ideen zugänglich und lassen sich oft manipulieren, aber in ihrem Hass gegen die Gesellschaftsnormen (bzw. die Gesellschaft als solche) können sie sehr gefährlich werden. Die jugendlichen Figuren in Glasers Werk haben meistens früh gelernt, Gewaltstrategien anzuwenden, um sich selbst gegen Unrecht zu behaupten. Die innere Stimme des Gewissens ist bei den meisten von ihnen mit der Zeit zurückgedrängt worden. Im erwachsenen Alter sind sie vielfach dafür anfällig, dem Rausch der Macht, Rache und Gewalt zu verfallen.

Im zweiten Teil des Beitrags habe ich Entmenschlichungsmechanismen dargestellt, die die Gewalt legitimieren oder selbst deren Spielarten sind. Die Ideologisierung mit ihrem Wahrheitsanspruch ist eines der mächtigsten Mittel dieser Art. In Geheimnis und Gewalt erscheinen die kommunistischen Ideale im Endeffekt oft als gewaltrechtfertigende Instrumente zur Beherrschung des Einzelnen. Glaser zeigt, wie rücksichtslos die Befehle der kommunistischen Zentralen sein konnten. Im Streben nach der Macht haben 
diese ihre Ideale selbst verleugnet, indem sie beispielsweise die französischen Kommunisten zu Morden an Deutschen (Kollektivschuld) aufforderten, wie in Geheimnis und Gewalt berichtet wird. Im Gegensatz zu ihren Idealen produziert auch die kommunistische Ideologie neue Machteliten. Zu weiteren Entmenschlichungsmechanismen, die Glaser beschreibt, gehört die Ausbeutung im Arbeitsprozess. Die Automatisierung in Betrieben hat beispielsweise die Einstellung der Arbeiter auf das Tempo des maschinellen Fließbands erfordert. Dadurch wurden die Arbeiter nicht nur ihrer menschlichen Würde beraubt, sondern auch der Lebensgefahr ausgesetzt. Der Nationalsozialismus mit seiner Rassenlehre ist wahrscheinlich die menschenverachtendste Ideologie überhaupt. Georg K. Glaser gelingt es in Geheimnis und Gewalt sehr überzeugend jene Mechanismen darzustellen, die die einstigen Opfer der Prügelpädagogik im großen Maße zu nationalsozialistischen Tätern machen konnten. Dabei wird das Konstrukt der Kollektivschuld der Deutschen vom Autor als ein Entmenschlichungsmechanismus verurteilt und als eine Art Fortsetzung des nationalsozialistischen Narrativs der einheitlichen Volksgemeinschaft entlarvt.

\section{Primärliteratur:}

Glaser, Georg K. (1989): Geheimnis und Gewalt. Ein Bericht. Frankfurt am Main: Stroemfeld/ Roter Stern.

Glaser, Georg K. (2007): Schluckebier und andere Erzählungen. Frankfurt am Main: Stroemfeld.

\section{Sekundärliteratur:}

Härtling, Peter (1966): Vergessene Bücher. Stuttgart: Goverts.

Krakauer, Siegfried (1985): Jugend in dieser Zeit. [Über Georg Glasers Schluckebier]. In: Georg K. Glaser: Aus der Chronik der Rosengasse und andere kleine Arbeiten. Berlin - Bonn: J.H.W. Dietz Nachf.

Rohrwasser, Michael (2007): Nachwort. In: Georg K. Glaser: Schluckebier und andere Erzählungen. Frankfurt am Main: Stroemfeld, S. 323-372.

Rohrwasser, Michael (1989): Nachwort. In: Georg K. Glaser: Geheimnis und Gewalt. Ein Bericht. Stroemfeld/ Roter Stern: Frankfurt am Main, S. 559-565.

Rohrwasser, Michael (1991): Georg K. Glaser: Die Partei und das Schreiben. In: Der Stalinismus und die Renegaten. Die Literatur der Exkommunisten. Stuttgart: Metzler.

Rühle, Otto (1922): Das proletarische Kind. München: Albert Langen.

Theweleit, Klaus (2019): Männerphantasien. Berlin: Matthes \& Seitz. 
Mgr. Pavel Knápek, Ph.D. / Pavel.Knapek@upce.cz

Univerzita Pardubice, Fakulta filozofická, Katedra cizích jazyků

Studentská 95, 53210 Pardubice, CZ

This work can be used in accordance with the Creative Commons BY-SA 4.0 International license terms and conditions (https://creativecommons.org/licenses/by-sa/4.0/legalcode). This does not apply to works or elements (such as image or photographs) that are used in the work under a contractual license or exception or limitation to relevant rights 